PROCEEDINGS OF THE

AMERICAN MATHEMATICAL SOCIETY

Volume 128, Number 10, Pages 3129-3136

S 0002-9939(00)05359-4

Article electronically published on April 7, 2000

\title{
HOWE CORRESPONDENCE FOR REAL UNITARY GROUPS II
}

\author{
ANNEGRET PAUL
}

(Communicated by Roe Goodman)

\begin{abstract}
A previous paper by the author describes the Howe correspondence for dual pairs of the form $(U(p, q), U(r, s))$ with $p+q=r+s$, in terms of Langlands parameters. We extend these results to the case $p+q=r+s+1$.
\end{abstract}

\section{INTRODUCTION}

Let $\left(G, G^{\prime}\right)$ be a reductive dual pair in $S p=S p(2 n, \mathbb{R})$, and let $\omega$ be the oscillator representation of the connected two-fold cover $\widetilde{S p}$ of $S p$. If $\widetilde{G}$ and $\widetilde{G}^{\prime}$ are the inverse images of $G$ and $G^{\prime}$ in $\widetilde{S p}$ by the covering map and $\pi$ and $\pi^{\prime}$ are irreducible admissible representations of $\widetilde{G}$ and $\widetilde{G}^{\prime}$, we say that $\pi$ corresponds to $\pi^{\prime}$ if (on the level of Harish-Chandra modules) $\pi \otimes \pi^{\prime}$ may be realized as a quotient of $\omega$. Howe $[\underline{H}$ proved that this correspondence defines a bijection between subsets of the genuine admissible duals of $\widetilde{G}$ and $\widetilde{G}^{\prime}$. In an earlier paper [P1] we consider dual pairs of the form $(U(p, q), U(r, s))$ with $p+q=r+s$ and determine the Howe correspondence explicitly, in terms of Langlands parameters. In this paper, we do the same for the case $p+q=r+s+1$.

Only genuine representations of the two-fold covers $\widetilde{U}(p, q)$ and $\widetilde{U}(r, s)$ (those which do not factor to $U(p, q)$ and $U(r, s))$ occur in the correspondence. These covers depend only on the parity of the rank of the opposite member of the dual pair. Fix $p$ and $q$, and let $\widetilde{U}(p, q)$ be the two-fold cover for $U(p, q)$ a member of any dual pair $(U(p, q), U(r, s))$ with $r+s+1=p+q$. If $\pi \in \widetilde{U}(p, q)_{\text {genuine }}$ corresponds to $\pi^{\prime} \in \widetilde{U}(r, s)_{\text {genuine }}$, we write $\theta_{r, s}(\pi)=\pi^{\prime}$ (the theta-lift of $\pi$ ). If $\pi$ does not occur in the correspondence, we write $\theta_{r, s}(\pi)=0$.

As in the equal rank case, we start with J.-S. Li's results on the correspondence of discrete series $[\mathrm{L}$ and make extensive use of the induction principle ([ $[\mathrm{K},[\mathrm{AB}]$ ) to determine the correspondence in general. This principle establishes that the Howe correspondence commutes with parabolic induction. Li found that every discrete series of $\widetilde{U}(p, q)$ whose Harish-Chandra parameter (in some obvious parametrization) contains 0 as one of its entries lifts to a discrete series of a certain $\widetilde{U}(r, s)$ of rank $p+q-1$. Starting from this result and using the induction principle and parabolic induction we show that a limit of discrete series with an odd number of 0's in its parameter (see Definition 3.1) lifts to a limit of discrete series (with an

Received by the editors October 15, 1998 and, in revised form, November 24, 1998.

2000 Mathematics Subject Classification. Primary 22E46.

Key words and phrases. Oscillator representation, reductive dual pairs, Langlands parameters. The author thanks the referee for several helpful suggestions. 
even number of 0's) of some $\widetilde{U}(r, s)$ of rank $p+q-1$. By persistence (Kudla), this representation will also lift to a limit of discrete series (even number of 0 's) of $\widetilde{U}(r+1, s+1)$. By switching the roles of $\widetilde{U}(p, q)$ and $\widetilde{U}(r+1, s+1)$ we now get the full correspondence for limits of discrete series. The general case then follows using the induction principle once more. In particular, we prove the following result.

Theorem 1.1. Let $\pi$ be a genuine irreducible admissible representation of $\widetilde{U}(p, q)$. There are two possibilities: either

(a) there are unique values $r$ and $s$ with $r+s+1=p+q$ such that $\theta_{r, s}(\pi) \neq 0$; or

(b) $\theta_{r, s}(\pi)=0$ for all choices of $r$ and $s$ with $r+s+1=p+q$, and there are unique values $r$ and $s$ with $r+s=p+q+1$ such that $\theta_{r, s}(\pi) \neq 0$ and $\theta_{r+1, s-1}(\pi) \neq 0$.

We give a precise condition in terms of the inducing data for the cases (a) and (b) in the theorem, and we identify the theta-lifts explicitly. This result gives additional evidence for the truth of the following conjecture on first occurrence (see also [KR]) which we prove in $\overline{\mathrm{P} 2}$ for a large family of representations including the discrete series.

Conjecture 1.2. Let $\pi$ be a genuine irreducible representation of a two-fold cover $\widetilde{U}(p, q)$ of $U(p, q)$. Then there are integers $r, s, r^{\prime}$, and $s^{\prime}$, with $r-s \neq r^{\prime}-s^{\prime}$ and $r+s+r^{\prime}+s^{\prime}=2 p+2 q+2$ such that $\theta_{r, s}(\pi) \neq 0$ and $\theta_{r^{\prime}, s^{\prime}}(\pi) \neq 0$.

An important tool for determining the correspondence for a dual pair $\left(G, G^{\prime}\right)$ is the correspondence of $K$ - and $K^{\prime}$-types ( $K, K^{\prime}$ are maximal compact subgroups of $\left.G, G^{\prime}\right)$ in the space of joint harmonics [H], which is subordinated to the dual pair correspondence. Howe assigns to each $K$-type a nonnegative integer, the degree, and there is the following relationship between the two correspondences.

Proposition 1.3 (Howe). Suppose $\pi$ corresponds to $\pi^{\prime}$ in the correspondence for $\left(G, G^{\prime}\right)$, and $\mu$ is a $K$-type of minimal degree in $\pi$. Then $\mu$ occurs in the space of joint harmonics and corresponds to a $K^{\prime}$-type $\mu^{\prime}$ of minimal degree in $\pi^{\prime}$.

The correspondence in the space of joint harmonics for dual pairs of the form $(U(p, q), U(r, s))$ is given in Lemma 1.4.5 of [P1]. In the equal rank case we found a nice compatibility between this minimality of Howe degree and the minimality of a $K$-type in the sense of Vogan. To a large extent, this compatibility extends to the present case. In particular, the correspondence for these dual pairs satisfies the following properties.

Proposition 1.4. Suppose $p+q=r+s+1$ and $\pi$ corresponds to $\pi^{\prime}$ in the Howe correspondence for the dual pair $(U(p, q), U(r, s))$.

(a) Each lowest $K$-type of $\pi$ is of minimal (Howe) degree in $\pi$ and corresponds to a lowest $K$-type of $\pi^{\prime}$ in the space of joint harmonics.

(b) $\pi$ is a discrete series representation or limit of discrete series if and only if $\pi^{\prime}$ is a discrete series or limit of discrete series. If $\pi$ is a discrete series representation, then so is $\pi^{\prime}$. Moreover, $\pi$ is tempered if and only if $\pi^{\prime}$ is.

\section{Preliminaries}

For the remainder of this paper, all two-fold covers of the form $\widetilde{U}(p, q)$ will be those determined by the dual pairs $(U(p, q), U(r, s))$ with $p+q=r+s+1$. Fix nonnegative integers $p$ and $q$. Let $\mathfrak{g}$ be the complexified Lie algebra of $\widetilde{U}(p, q), K$ 
a maximal compact subgroup, and $\mathfrak{t}_{0}$ a Cartan subalgebra of the Lie algebra $\mathfrak{k}_{0}$ of $K$. The compact roots in $\Delta(\mathfrak{g}, \mathfrak{t})$ are (with obvious notation) $\left\{e_{i}-e_{j}: 1 \leq\right.$ $i, j \leq p\} \cup\left\{f_{i}-f_{j}: 1 \leq i, j \leq q\right\}$. We fix a positive system of compact roots $\Delta_{c}^{+}$by requiring $i<j$. The noncompact roots are $\left\{ \pm\left(e_{i}-f_{j}\right): 1 \leq i \leq p, 1 \leq\right.$ $j \leq q\}$. Langlands parameters of genuine irreducible admissible representations of $\widetilde{U}(p, q)$ are described in [P1] (see also [V]). Recall that every such representation may be realized as the unique irreducible quotient of a standard representation $\operatorname{Ind} d_{P}^{\widetilde{U}(p, q)}(\rho \otimes \chi \otimes \mathbb{1})$, where $P=M N$ is a cuspidal parabolic subgroup of $\widetilde{U}(p, q)$ with Levi factor $M \cong \widetilde{U}(p-t, q-t) \times\left(\mathbb{C}^{\times}\right)^{t}$ for some $t, \rho$ is a genuine limit of discrete series representation of $\widetilde{U}(p-t, q-t)$, and $\chi$ is a character of $\left(\mathbb{C}^{\times}\right)^{t}$. Genuine limit of discrete series representations are parametrized by pairs $(\lambda, \Psi)$, where $\lambda \in i \mathbf{t}_{0}^{*}$ is of the form

$$
\lambda=(\underbrace{a_{1}, \ldots, a_{1}}_{k_{1}}, \underbrace{a_{2}, \ldots, a_{2}}_{k_{2}}, \ldots, \underbrace{a_{x}, \ldots, a_{x}}_{k_{x}} ; \underbrace{a_{1}, \ldots, a_{1}}_{l_{1}}, \underbrace{a_{2}, \ldots, a_{2}}_{l_{2}}, \ldots, \underbrace{a_{x}, \ldots, a_{x}}_{l_{x}})
$$

with $a_{i} \in \mathbb{Z}, a_{1}>a_{2}>\cdots>a_{x}, p=\sum_{i} k_{i}, q=\sum_{i} l_{i}$, and $\Psi$ is a system of positive roots in $\Delta(\mathfrak{g}, \mathfrak{t})$ containing $\Delta_{c}^{+}$. Moreover, $\lambda$ and $\Psi$ satisfy the following conditions: $\left|k_{i}-l_{i}\right| \leq 1$ for all $i, \lambda$ is dominant for $\Psi$, and if $\alpha \in \Psi$ is simple and $\langle\alpha, \lambda\rangle=0$, then $\alpha$ is noncompact.

The term K-type will always refer to an equivalence class of irreducible representations of a maximal compact subgroup of the group under consideration. In addition, we will use the following

Notation 2.2. (a) For $n$ a positive integer, let $\mu=\left(\mu_{1}, \mu_{2}, \ldots, \mu_{n}\right)$ be an $n$-tuple of integers, and $\nu=\left(\nu_{1}, \nu_{2}, \ldots, \nu_{n}\right)$ an $n$-tuple of complex numbers. Then $\chi(\mu, \nu)$ is the character of $\left(\mathbb{C}^{\times}\right)^{n}$ given by

$$
\left(r_{1} e^{i \theta_{1}}, \ldots, r_{n} e^{i \theta_{n}}\right) \mapsto \prod_{i=1}^{n} r_{i}^{\nu_{i}} e^{i \mu_{i} \theta_{i}}
$$

(b) For $k \leq \min \{p, q\}$ let $P=M N$ be a cuspidal parabolic subgroup of $\widetilde{U}(p, q)$ with Levi factor $M \cong \widetilde{U}(p-k, q-k) \times\left(\mathbb{C}^{\times}\right)^{k}$. For $\rho$ a discrete series or limit of discrete series representation of $\widetilde{U}(p-k, q-k)$ and for $\chi=\chi(\mu, \nu)$ a character of $\left(\mathbb{C}^{\times}\right)^{k}$, we let $I(p, q, k, \rho, \mu, \nu)$ denote the standard representation Ind $\widetilde{U}(p, q)(\rho \otimes \chi \otimes \mathbb{1})$. We are using normalized induction. Notice that the composition series of $I(p, q, k, \rho, \mu, \nu)$ is uniquely defined.

In this paper, we will use many of the results of [P1] and some of [P2]. In the interest of brevity we only restate two of them here.

Since $\widetilde{U}(p, q)$ and $\widetilde{U}(q, p)$ are naturally isomorphic, any $\pi \in \widetilde{U}(p, q) \widehat{\text { genuine }}$ may be considered in a natural way as a representation of $\widetilde{U}(q, p)$. The behavior of the Howe correspondence with respect to this identification is given by the following result from [P1].

Proposition 2.3 (Symmetry). Let $\pi \in \widetilde{U}(p, q)_{\text {genuine }}, \pi^{\prime} \in \widetilde{U}(r, s)_{\text {genuine }}$, and let $\pi^{*}$ and $\pi^{\prime *}$ be the contragredient representations of $\pi$ and $\pi^{\prime}$ respectively. Then

(a) $\theta_{r, s}(\pi)=\pi^{\prime}$ if and only if $\theta_{s, r}\left(\pi^{*}\right)=\pi^{* *}$;

(b) $\pi \leftrightarrow \pi^{\prime}$ in the correspondence for the dual pair $(U(p, q), U(r, s))$ if and only if $\pi \leftrightarrow \pi^{\prime}$ in the correspondence for the dual pair $(U(q, p), U(s, r))$. 
The next result which is in [P2] is useful for proving non-occurrence.

Proposition 2.4. Let $\pi \in \widetilde{U}(p, q)_{\text {genuine }}$. Suppose $r, s, r^{\prime}$, and $s^{\prime}$ are nonnegative integers such that $r-s \neq r^{\prime}-s^{\prime}$. If $\theta_{r, s}(\pi) \neq 0$ and $\theta_{r^{\prime}, s^{\prime}}(\pi) \neq 0$, then

$$
r+s+r^{\prime}+s^{\prime} \geq 2 p+2 q+2 \text {. }
$$

\section{THE CORRESPONDENCE}

Definition 3.1. Let $(\lambda, \Psi)$ be the parameters of a genuine limit of discrete series representation $\pi$ of $\widetilde{U}(p, q)$. If

$$
\lambda=(a_{1}, a_{2}, \ldots, a_{k}, \underbrace{0, \ldots, 0}_{z}, b_{1}, \ldots, b_{l} ; c_{1}, \ldots, c_{m}, \underbrace{0, \ldots, 0}_{w}, d_{1}, \ldots, d_{n})
$$

with $a_{1} \geq a_{2} \geq \cdots \geq a_{k}>0>b_{1} \geq \cdots \geq b_{l}$ and $c_{1} \geq \cdots \geq c_{m}>0>d_{1} \geq \cdots \geq$ $d_{n}$, assume that

$$
z+w \geq 1
$$

Let

$$
(u, v)= \begin{cases}(w, z-1) & \text { if } z>w \text { or } e_{k+1}-f_{m+1} \in \Psi \\ (w-1, z) & \text { if } z<w \text { or } f_{m+1}-e_{k+1} \in \Psi\end{cases}
$$

$r(\lambda, \Psi)=k+n+u$ and $s(\lambda, \Psi)=m+l+v$.

We define the representation $\Gamma \pi$ of $\widetilde{U}(r(\lambda, \Psi), s(\lambda, \Psi))$ to be the limit of discrete series representation with the parameters $(\Gamma \lambda, \Gamma \Psi)$ given by

$$
\Gamma \lambda=(a_{1}, a_{2}, \ldots, a_{k}, \underbrace{0, \ldots, 0}_{u}, d_{1}, \ldots, d_{n} ; c_{1}, \ldots, c_{m}, \underbrace{0, \ldots, 0}_{v}, b_{1}, \ldots, b_{l}),
$$

and $\Gamma \Psi$ the unique system of positive roots satisfying the conditions following (2.1) and

(a) if $i \leq k, j \leq m$, then $e_{i}-f_{j} \in \Gamma \Psi \Leftrightarrow e_{i}-f_{j} \in \Psi$;

(b) if $i>k+u, j>m+v$, then $e_{i}-f_{j} \in \Gamma \Psi \Leftrightarrow f_{i+m+w-k-u}-e_{j+k+z-m-v} \in \Psi$;

(c) if $u>0$ and $v>0$, then $e_{k+1}-f_{m+1} \in \Gamma \Psi \Leftrightarrow e_{k+1}-f_{m+1} \in \Psi$.

We are now ready to state our main result.

Theorem 3.4 (Main Theorem). Let $\pi$ be a genuine irreducible representation of $\widetilde{U}(p, q)$. Realize $\pi$ as the unique irreducible quotient of a standard representation $I(p, q, t, \rho, \mu, \nu)$ as in Chapter 3 of [P1]. Let $(\lambda, \Psi)$ be the parameters of the genuine limit of discrete series representation $\rho$ of $\widetilde{U}(p-t, q-t)$, and write $\lambda$ as in (3.2).

(a) If $\lambda$ satisfies (3.3), let $r=r(\lambda, \Psi)+t$ and $s=s(\lambda, \Psi)+t$. Then $\theta_{r, s}(\pi)$ is the unique lowest $K$-type constituent of the standard representation $I(r, s, t, \Gamma \rho, \mu, \nu)$, and $\theta_{r^{\prime}, s^{\prime}}(\pi)=0$ for all $r^{\prime}$ and $s^{\prime}$ with $r^{\prime}+s^{\prime}+1=p+q$ and $r^{\prime} \neq r$.

(b) If $\lambda$ does not satisfy (3.3), then $\theta_{r, s}(\pi)=0$ for all integers $r$ and $s$ such that $r+s+1=p+q$.

(c) For case (b), let $r_{1}=k+n+t+1, s_{1}=l+m+t, r_{2}=r_{1}-1$, and $s_{2}=s_{1}+1$. Then for $i=1,2, \theta_{r_{i}, s_{i}}(\pi)=\pi_{i}$, where $\pi_{i}$ is the unique lowest $K$-type constituent of $I\left(r_{i}, s_{i}, t, \rho_{i}, \mu, \nu\right)$ given by the following data:

$$
\begin{aligned}
& \lambda_{1}=\left(a_{1}, \ldots, a_{k}, 0, d_{1}, \ldots, d_{n} ; c_{1}, \ldots, c_{m}, b_{1}, \ldots, b_{l}\right), \\
& \lambda_{2}=\left(a_{1}, \ldots, a_{k}, d_{1}, \ldots, d_{n} ; c_{1}, \ldots, c_{m}, 0, b_{1}, \ldots, b_{l}\right),
\end{aligned}
$$


$\Psi_{i}$ is the unique system of positive roots for $\lambda_{i}$ such that $\Gamma \Psi_{i}=\Psi$, and $\rho_{i}$ the limit of discrete series representation of $\widetilde{U}\left(r_{i}-t, s_{i}-t\right)$ with parameters $\left(\lambda_{i}, \Psi_{i}\right)$.

Proof. The proof of Theorem 3.4 is similar to the proof of Theorem 6.1 of [P1. We outline the main steps.

First notice that by Proposition 2.4, any representation $\pi$ of $\widetilde{U}(p, q)$ may lift to at most one unitary group of rank $p+q-1$. Also, (c) will follow from (a) since $\rho=\Gamma \rho_{i}, r=r\left(\lambda_{i}, \Psi_{i}\right)+t$, and $s=s\left(\lambda_{i}, \Psi_{i}\right)+t$. Since in this case $\pi$ occurs as the theta lift of representations of two different unitary groups which are both of rank $p+q+1$, part (b) will follow using Proposition 2.4. It remains to prove the first part of (a).

If $\pi$ is a discrete series representation, then this follows from Theorem 6.2 of [L].

Now assume that $\pi$ is a limit of discrete series representation with parameters $(\lambda, \Psi)$ as in Definition 3.1, and consider first the case that $|z-w|=1$. By symmetry (Proposition 2.3) we may assume that $z=w+1$. Then $\pi$ may be realized as a summand of a standard representation $I\left(p, q, t^{\prime}, \rho, \mu, 0\right)$ as in Lemma 3.2.6 of [P1, where $\rho$ is a discrete series representation with Harish-Chandra parameter $\lambda_{d}$ of the form

$$
\lambda_{d}=\left(x_{1}, x_{2}, \ldots, x_{d}, 0, y_{1}, \ldots, y_{e} ; z_{1}, \ldots, z_{f}, w_{1}, \ldots, w_{g}\right)
$$

with $x_{d}>0>y_{1}, z_{f}>0>w_{1}, d+e+1=p-t^{\prime}, f+g=q-t^{\prime}, d+g=r-t^{\prime}$, and $e+f=s-t^{\prime}$. By Theorem 6.2 of $\left[\mathrm{L}, \theta_{r-t^{\prime}, s-t^{\prime}}(\rho)\right.$ is the discrete series representation $\rho^{\prime}$ of $\widetilde{U}\left(r-t^{\prime}, s-t^{\prime}\right)$ with Harish-Chandra parameter

$$
\left(x_{1}, x_{2}, \ldots, x_{d}, w_{1}, \ldots, w_{g} ; z_{1}, \ldots, z_{f}, y_{1}, \ldots, y_{e}\right) .
$$

Using the induction principle (Theorem 4.5.5 of [P1]) we know there is a nonzero $\widetilde{U}(p, q) \times \widetilde{U}(r, s)$-map

$$
\omega \longrightarrow \operatorname{Ind}_{P}^{\widetilde{U}(p, q)}\left(\rho \otimes \sigma_{\mu, 0} \otimes \mathbb{1}\right) \otimes \operatorname{Ind}_{P^{\prime}}^{\widetilde{U}(r, s)}\left(\rho^{\prime} \otimes \sigma_{\mu, 0} \otimes \mathbb{1}\right) .
$$

Here $P=M N$ and $P^{\prime}=M^{\prime} N^{\prime}$ are parabolic subgroups of $\widetilde{U}(p, q)$ and $\widetilde{U}(r, s)$ with Levi factors $M \cong \widetilde{U}\left(p-t^{\prime}, q-t^{\prime}\right) \times G L\left(t^{\prime}, \mathbb{C}\right)$ and $M^{\prime} \cong \widetilde{U}\left(r-t^{\prime}, s-t^{\prime}\right) \times G L\left(t^{\prime}, \mathbb{C}\right)$ respectively, and $\sigma_{\mu, 0}$ is the irreducible representation of $G L\left(t^{\prime}, \mathbb{C}\right)$ associated to the parameters $(\mu, 0)$ as in Lemma 5.2.6 of [P1]. The lowest $U\left(t^{\prime}\right)$-type $\delta$ of this representation has a highest weight which is Weyl group conjugate to $\mu$. Since the induced representations in (3.6) are tempered, they are independent of the choice of $N$ and $N^{\prime}$, and equivalent to $I\left(p, q, t^{\prime}, \rho, \mu, 0\right)$ and $I\left(r, s, t^{\prime}, \rho^{\prime}, \mu, 0\right)$ respectively. It is easy to see that $\Gamma \pi$ is a summand of the second of these standard representations. To prove that $\theta_{r, s}(\pi)=\Gamma \pi$, we now show that $\gamma \otimes \gamma^{\prime}$ is in the image of the map (3.6), where $\gamma$ and $\gamma^{\prime}$ are the lowest $K$-types of $\pi$ and $\Gamma \pi$ respectively. (Recall that they have multiplicity one in the standard representations.) Using Theorem 4.6.6 of [P1], this now follows from Lemma 5.4.4 of [P1], and Proposition 3.7 and Lemma 3.10 below.

Proposition 3.7. Let $\pi=\pi(\lambda, \Psi)$ be a genuine limit of discrete series representation of $\widetilde{U}(p, q)$ satisfying (3.3). Let $r=r(\lambda, \Psi), s=s(\lambda, \Psi)$, and $\pi^{\prime}=\Gamma \pi$. Then the lowest $K$-types $\gamma$ and $\gamma^{\prime}$ of $\pi$ and $\pi^{\prime}$ are harmonic and of minimal degree for the dual pair $(U(p, q), U(r, s))$ and correspond in the space of joint harmonics.

Proof. By symmetry (Proposition 2.3) it is sufficient to consider the case $z>w$ or $e_{k+1}-f_{m+1} \in \Psi$ so that $z \geq w$. Let $\lambda^{\prime}=\Gamma \lambda$ and $\Psi^{\prime}=\Gamma \Psi$. The highest weight 
of $\gamma$ is given by $\Lambda=\lambda+\rho_{n}-\rho_{c}$, where $\rho_{n}$ and $\rho_{c}$ are one half the sums of the noncompact and compact roots in $\Psi$ respectively. Similarly, $\Lambda^{\prime}=\lambda^{\prime}+\rho_{n}^{\prime}-\rho_{c}^{\prime}$ is the highest weight of $\gamma^{\prime}$, with $\rho_{n}^{\prime}$ and $\rho_{c}^{\prime}$ defined analogously. Write $\lambda$ as in (3.2). Let

$$
\begin{aligned}
& \alpha_{i}=\sharp\left\{j \leq m: e_{i}-f_{j} \in \Psi\right\}, \text { for } 1 \leq i \leq k, \\
& \beta_{i}=\sharp\left\{j \leq n: e_{k+z+i}-f_{m+w+j} \in \Psi\right\}, \text { for } 1 \leq i \leq l, \\
& \gamma_{i}=\sharp\left\{j \leq k: f_{i}-e_{j} \in \Psi\right\}, \text { for } 1 \leq i \leq m, \\
& \delta_{i}=\sharp\left\{j \leq l: f_{m+w+i}-e_{k+z+j} \in \Psi\right\}, \text { for } 1 \leq i \leq n .
\end{aligned}
$$

If $z=w+1$, then $u=v=w, r-s=k+n-m-l$, and

$$
\Lambda=\left(A_{1}, \ldots, A_{k}, Z_{1}, \ldots, Z_{z}, B_{1}, \ldots, B_{l} ; C_{1}, \ldots, C_{m}, \tilde{Z}_{1}, \ldots, \tilde{Z}_{w}, D_{1}, \ldots, D_{n}\right),
$$

with

$$
\begin{aligned}
A_{i} & =a_{i}+\alpha_{i}-\frac{n-m-l-k}{2}+i-1=\frac{r-s}{2}+a_{i}+\alpha_{i}-k+i-1, \\
Z_{i} & =\frac{n-m-l+k}{2}=\frac{r-s}{2} \\
B_{i} & =b_{i}+\beta_{i}+\frac{k-l-m-n}{2}+i=\frac{r-s}{2}+b_{i}+\beta_{i}-n+i, \\
C_{i} & =c_{i}+\gamma_{i}+\frac{l-k-n-m}{2}+i=\frac{s-r}{2}+c_{i}+\gamma_{i}-m+i, \\
\tilde{Z}_{i} & =\frac{l-k-n+m}{2}=\frac{s-r}{2} \\
D_{i} & =d_{i}+\delta_{i}+\frac{m-k-l-n}{2}+i-1=\frac{s-r}{2}+d_{i}+\delta_{i}-l+i-1 .
\end{aligned}
$$

Similarly,

$$
\Lambda^{\prime}=\left(A_{1}^{\prime}, \ldots, A_{k}^{\prime}, Z_{1}^{\prime}, \ldots, Z_{w}^{\prime}, D_{1}^{\prime}, \ldots, D_{n}^{\prime} ; C_{1}^{\prime}, \ldots, C_{m}^{\prime}, \tilde{Z}_{1}^{\prime}, \ldots, \tilde{Z}_{w}^{\prime}, B_{1}^{\prime}, \ldots, B_{l}^{\prime}\right),
$$

with

$$
\begin{aligned}
A_{i}^{\prime} & =\frac{p-q}{2}+a_{i}+\alpha_{i}-k+i-1, \\
Z_{i}^{\prime} & =\frac{p-q}{2} \\
D_{i}^{\prime} & =\frac{p-q}{2}+d_{i}+\delta_{i}-l+i-1, \\
C_{i}^{\prime} & =\frac{q-p}{2}+c_{i}+\gamma_{i}-m+i, \\
\tilde{Z}_{i}^{\prime} & =\frac{q-p}{2} \\
B_{i}^{\prime} & =\frac{q-p}{2}+b_{i}+\beta_{i}-n+i .
\end{aligned}
$$

By Lemma 1.4.5 of [P1], $\gamma$ and $\gamma^{\prime}$ are harmonic and correspond.

The proof of the fact that $\gamma$ is of minimal degree in $\pi$ is similar to the proofs of the analogous facts in Proposition 5.1.4. of [P1] and Lemma 4.2 of [P2].

The case $z=w$, with $u=w, v=w-1$ and $r-s=k+n+1-m-l$ is similar.

Lemma 3.10. In the setting of (3.6), let $\eta$ be the minimal $K$-type of the discrete series representation $\rho$. The restriction of $\gamma$ to $\widetilde{U}\left(p-t^{\prime}\right) \times \widetilde{U}\left(q-t^{\prime}\right) \times U\left(t^{\prime}\right)$ contains $\eta \otimes \delta$. Moreover, $\eta \otimes \delta$ is of minimal degree in $\rho \otimes \sigma_{\mu, 0}$ for the dual pair $\left(U\left(p-t^{\prime}, q-t^{\prime}\right) \times G L\left(t^{\prime}, \mathbb{C}\right), U\left(r-t^{\prime}, s-t^{\prime}\right) \times G L\left(t^{\prime}, \mathbb{C}\right)\right)$, and $\operatorname{deg}(\eta \otimes \delta)=$ $\operatorname{deg}(\gamma)$. 
Proof. It is straightforward to see that the highest weight of $\eta \otimes \delta$ is obtained by restricting the highest weight of $\gamma$, which implies the first part of the lemma. The equality of degrees then follows as in Lemma 5.2.8 of [P1]. By Proposition 3.7, $\eta$ is of minimal degree in $\rho$ for the dual pair $\left(U\left(p-t^{\prime}, q-t^{\prime}\right), U\left(r-t^{\prime}, s-t^{\prime}\right)\right)$, and $\delta$ is of minimal degree in $\sigma_{\mu, 0}$ for the dual pair $\left(G L\left(t^{\prime}, \mathbb{C}\right), G L\left(t^{\prime}, \mathbb{C}\right)\right)$ by Lemma 4.1 of AB. It follows that $\eta \otimes \delta$ is of minimal degree in $\rho \otimes \sigma_{\mu, 0}$.

Now assume that $z=w \geq 1$, and consider the representation $\pi^{\prime}=\Gamma \pi$ of $\widetilde{U}(r, s)$. Notice that this representation is a limit of discrete series representation of the form considered above, so that we know that $\theta_{p-1, q-1}\left(\pi^{\prime}\right)=\Gamma \pi^{\prime}$. By Theorem 4.5.5 of [P1], $\theta_{p, q}\left(\pi^{\prime}\right)$ is a constituent of $I\left(p, q, 1, \Gamma \pi^{\prime}, 0,0\right)$. This representation is a sum of limit of discrete series representations, one of which is easily seen to be $\pi$. The fact that $\theta_{p, q}\left(\pi^{\prime}\right)=\pi$ now follows using Proposition 3.7.

For the general case assume that $\pi$ is realized as the unique irreducible quotient of a standard representation $I(p, q, t, \rho, \mu, \nu)$ as in the statement of the theorem, with $\rho=\rho(\lambda, \Psi)$ a genuine limit of discrete series representation of $\widetilde{U}(p-t, q-t)$ satisfying (3.3). One can easily check using the conditions in $\nabla]$ that $I(r, s, t, \Gamma \rho, \mu, \nu)$ has a unique lowest $K$-type constituent $\pi^{\prime}$ as well. Since $\rho$ corresponds to $\Gamma \rho$ in the correspondence for the dual pair $(U(p-t, q-t), U(r-t, s-t))$ (by the first part of the proof), Theorem 4.5.5 of [P1] implies that for some parabolic subgroups $P=M N$ and $P^{\prime}=M^{\prime} N^{\prime}$ of $\widetilde{U}(p, q)$ and $\widetilde{U}(r, s)$ with Levi factors $M \cong \widetilde{U}(p-t, q-t) \times G L(t, \mathbb{C})$ and $M^{\prime} \cong \widetilde{U}(r-t, s-t) \times G L(t, \mathbb{C})$, there is a nonzero $\widetilde{U}(p, q) \times \widetilde{U}(r, s)$ map

$$
\omega \longrightarrow \operatorname{Ind}_{P}^{\widetilde{U}(p, q)}\left(\rho \otimes \sigma_{\mu, \nu} \otimes \mathbb{1}\right) \otimes \operatorname{Ind}_{P^{\prime}}^{\widetilde{U}(r, s)}\left(\Gamma \rho \otimes \sigma_{\mu,-\nu} \otimes \mathbb{1}\right) .
$$

The representations $\pi$ and $\pi^{\prime}$ are the unique lowest $K$-type constituents of the induced representations in (3.11). (Recall that $I(r, s, t, \Gamma \rho, \mu, \nu)$ and $I(r, s, t, \Gamma \rho, \mu,-\nu)$ have the same composition series.) Moreover, we can arrange that either of these constituents is a quotient of the respective induced representation (see the proof of Theorem 6.1 of [P1]). In order to complete the proof of Theorem 3.4, we will show that there are lowest $K$-types $\eta$ and $\eta^{\prime}$ of $\pi$ and $\pi^{\prime}$ (necessarily with multiplicity one in the induced representations) which correspond in the space of joint harmonics and such that $\eta \otimes \eta^{\prime}$ is in the image of the map (3.11). Using Theorem 4.6.6 of [P1], this follows from Proposition 3.12 and Lemma 3.14 below.

Proposition 3.12. In the setting of Theorem 3.4, assume the parameter $\lambda$ of $\rho$ is of the form (3.2) with $|w-z|=1$. Let $\eta$ be a lowest $K$-type of $I=I(p, q, t, \rho, \mu, \nu)$. Then $\eta$ is of minimal degree in I and harmonic for the dual pairs $(U(p, q), U(r, s))$ and $(U(p, q), U(r+1, s+1))$. The $K$-types $\eta^{\prime}$ and $\eta^{\prime \prime}$ which correspond to $\eta$ in the respective spaces of joint harmonics for these dual pairs have the same Vogan norm as the lowest $K$-types of $I(r, s, t, \Gamma \rho, \mu, \nu)$ and $I\left(r+1, s+1, t, \rho^{\prime}, \mu, \nu\right)$, where $\rho^{\prime}$ is the (unique) limit of discrete series representation of $\widetilde{U}(r+1-t, s+1-t)$ with $\Gamma \rho^{\prime}=\rho$.

Proof. Similar to the proofs of Proposition 5.1.4 and Lemma 5.3.1 of [P1]. We omit the details.

Remark 3.13. It is also true that every lowest $K$-type of $I\left(r+1, s+1, t, \rho^{\prime}, \mu, \nu\right)$ is of minimal degree and corresponds to a lowest $K$-type of $I$ in the space of joint harmonics for the dual pair $(U(r+1, s+1), U(p, q))$ (see Proposition 1.4). 
Lemma 3.14. In the setting of Proposition 3.12, let $\gamma$ be the lowest $K$-type of $\rho$, and $\delta$ the lowest $U(t)$-type of $\sigma_{\mu, \nu}$. Then the restriction of $\eta$ to $\widetilde{U}(p-t) \times \widetilde{U}(q-t) \times$ $U(t)$ contains $\gamma \otimes \delta$. Moreover, $\gamma \otimes \delta$ is of minimal degree in $\rho \otimes \sigma_{\mu, \nu}$ and $\operatorname{deg}(\eta)=$ $\operatorname{deg}(\gamma \otimes \delta)$ for both the dual pairs $(U(p-t, q-t) \times G L(t, \mathbb{C}), U(r-t, s-t) \times G L(t, \mathbb{C}))$ and $(U(p-t, q-t) \times G L(t, \mathbb{C}), U(r+1-t, s+1-t) \times G L(t, \mathbb{C}))$.

Proof. As in the proof of Lemma 3.10, one shows that the highest weight of $\gamma \otimes \delta$ may be obtained by restriction of the highest weight of $\eta$, which is straightforward and implies the first part of the lemma. The equality of degrees is then easily confirmed by a calculation as in Lemma 5.3 .3 of [P1]. The remaining part of the statement then follows using Lemma 4.1 of $\mathrm{AB}$ and Proposition 3.7.

This completes the proof of Theorem 3.4.

\section{REFERENCES}

[AB] J. Adams and D. Barbasch, Dual pair correspondence for complex groups, J. Func. Anal. 132 (1) (1995), 1-42. MR 96h:22003

[H] R. Howe, Transcending classical invariant theory, J. of the Am. Math. Soc. 2 (3) (July 1989), 535-552. MR 90k:22016

[K] S. Kudla, On the local theta correspondence, Invent. Math. 83 (1986), 229-255. MR 87e:22037

[KR] S. Kudla and S. Rallis, First occurrence in the theta correspondence, Notes for a 20 minute talk at the AMS Meeting at Northeastern University, October 8, 1995.

[L] J.-S. Li, Local theta lifting for unitary representations with non-zero cohomology, Duke Math. J. 61 (1990), 913-937. MR 92f:22024

[P1] A. Paul, Howe correspondence for real unitary groups, J. Funct. Anal. 159 (1998), no. 2, 384-431. CMP 99:04

[P2] A. Paul, First occurrence for the dual pairs $(U(p, q), U(r, s))$, Canad. J. Math. 51 (3) (1999), 636-657. CMP 99:16

[V] D. Vogan, Unitarizibility of certain series of representations, Ann. of Math. 120 (1984), 141-187. MR 86h:22028

Department of Mathematics, University of California, Berkeley, California 947203840

E-mail address: apaul@math.berkeley.edu

Current address: Department of Mathematics \& Statistics, Western Michigan University, Kalamazoo, Michigan 49008-5152

E-mail address: paula@wmich.edu 